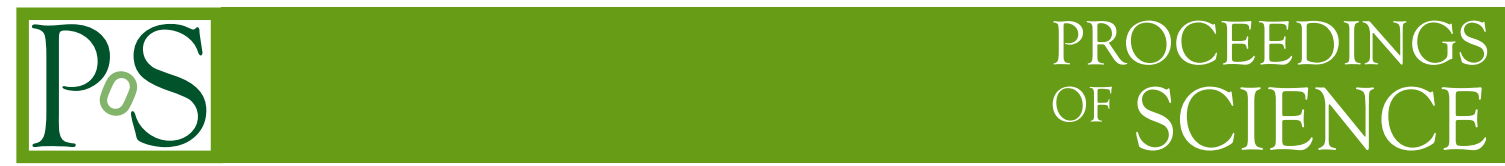

\title{
Semileptonic decays at B-Factories
}

\author{
Franco Simonetto*t \\ INFN \& Università di Padova \\ E-mail: franco.simonettoepd.infn.it
}

Semileptonic B decays provide valuable informations on some fundamental parameters of the standard model of the electroweak interactions, as the CKM parameters $\left|V_{c b}\right|$ and $\left|V_{u b}\right|$. I present here a comprehensive review, with a focus on still unsolved issues

The International Conference on B-Physics at Frontier Machines - BEAUTY2018

6-11 May, 2018

La Biodola, Elba Island, Italy

${ }^{*}$ Speaker.

${ }^{\dagger}$ A footnote may follow. 


\section{Introduction}

The couplings of the $b$ quark to the lighter partners $u$ and $c$ are expressed by the elements $\left|V_{u b}\right|$ and $\left|V_{c b}\right|$ of the Cabibbo Kobayashi Maskawa matrix, the long lifetime of B hadrons being a direct consequence of their small size. They are both cornerstones of flavor Physics: $\left|V_{c b}\right|$ sets the scale for a unitarity triangle of the CKM and the ratio $\left|V_{u b}\right| /\left|V_{c b}\right|$ measures the length of the side opposite to the angle $\phi_{1}$ in that triangle; CP violation in the evolution and decay of neutral Kaons also depends on $\left|V_{c b}\right|$. For all these reasons, a precise determination of these parameters is demanded in the exploration of $\mathrm{CP}$ violation in the hadron sector.

The best measurements of both parameters are obtained from B hadrons semileptonic decays $b \rightarrow \ell^{-} \bar{v}_{\ell} X$, where $\ell$ stands for either electron $(e)$ or muon $(\mu)$. The large branching fractions and the clear experimental signature allow the collection of large data sets with relatively small, well controlled background. The lepton and the hadron currents factorize; the hadron current is decomposed in terms of perturbative QCD contributions and non perturbative terms which are computed with tecniques inspired by Heavy Quark Symmetry [1],[2], an approximation of QCD in the limit of very large hadron masses.

Several different measurements are in fact performed. In some cases, all the observable particles produced in the final state are reconstructed, the unobserved neutrino properties are then inferred using constraints form energy-momentum conservation: these are referred to as "Exclusive SemiLeptonic decays" (ESL). In a more inclusive approach ("Inclusive SemiLeptonic",ISL) only some of the decay products are used to tag and characterize the events, the charged lepton and possibly some of the final state hadrons. Different computation schemes are required for ESL and ISL.

The large amount of events collected at the B-factories (about 470 million $e^{+} e^{-} \rightarrow B \bar{B}$ collisions were collected by $B A B A R$ at PEPII and about 770 million by Belle at KEK) allows for "tagged analysis", where the semileptonic decay is searched for in events where the other B meson is entirely reconstructed in a hadronic final state (more than one thousand different decay modes are exploited): this improves the signal to noise ratio and the constraints on the neutrino momentum, at the price of a considerable reduction of the event rate (tagging efficiency ranges between three and five per mill).

By combining many different results, impressive precision is achieved for $\left|V_{c b}\right|, \mathrm{O}(1 \%)$, and $\left|V_{u b}\right|$ (better than $10 \%$ ). Nevertheless the results from ESL determinations are at odds with those from ISL for both parameters.

The tagged techniques developed for $\left|V_{c b}\right|$ measurements allow for the much more difficult measurement of the ESL $B \rightarrow \tau^{-} \bar{v}_{\tau} D^{(*)}$. The combined results from BABAR and Belle miss the standard model expectation by about four standard deviations, opening a possible breach for new Physics effects in the heavy fermions sector.

\section{2. $\left|V_{c b}\right|$ in ISL decays}

The partial decay width for the inclusive semileptonic decay $b \rightarrow c \ell \bar{v}_{\ell}$ decay can be expressed as the product of $\left|V_{c b}\right|$ with a "spectator" term and other factors which include electroweak 
Table 1: results of the fits to lepton and hadron moments from ISL in two different OPE expansions

\begin{tabular}{l|c|c|c|c}
\hline scheme & $\mathscr{B}\left(b \rightarrow c \ell \bar{v}_{\ell}\right)$ & $\left|V_{c b}\right| \times 10^{3}$ & $m_{b}(\mathrm{GeV})$ & $\mu_{\pi}^{2} \simeq-\lambda_{1}(\mathrm{GeV})$ \\
\hline kinetic & $10.65 \pm 0.16$ & $42.19 \pm 0.73$ & $4.554 \pm 0.018$ & $0.464 \pm 0.076$ \\
$1 \mathrm{~S}$ & $10.65 \pm 0.16$ & $41.98 \pm 0.45$ & $4.691 \pm 0.037$ & $0.362 \pm 0.076$ \\
\hline
\end{tabular}

$(\mathscr{O}(1 \%))$ and QCD corrections (see [3]):

$$
\Gamma_{b \rightarrow \ell^{-} \bar{v}_{\ell} c}=\left|V_{c b}\right|^{2} \times \frac{G_{F}^{2} m_{b}^{5}(\mu)}{192 \pi^{3}} \times\left(1+\mathscr{A}_{e w k}\right) \times \mathscr{A}_{Q C D}\left(\frac{\alpha_{s}(\mu)}{\pi}, \frac{\mathscr{M}_{j}}{m_{b}(\mu)}, r=\frac{m_{c}}{m_{b}}(\mu)\right) .
$$

These last are expressed as the combination of perturbative terms, computed up to second order in $\frac{\alpha_{s}}{\pi}$, and an Operator Product Expansion of the non perturbative part, a power series of $\frac{\mathscr{M}_{j}}{m_{b}}$, where $\mathscr{M}_{j}$ are the expectation values of several low energy operators, such as the $b$ quark kinetic energy or its chromo-magnetic moment: only powers equal to or smaller than $m_{b}^{-2}$ compare in the expansion, easing convergence. Different expansion schemes have been proposed, for a detailed review see for instance [4]. Measurements of the spectra of the decay products help constrain the non-perturbative parameters, allowing a precise measurement of $\left|V_{c b}\right|$ : to dilute the nuisance induced by intermediate hadronic resonances, moments of the distributions of the electron energy spectrum (up to the forth power) and of the hadronic mass spectrum (up to the third power) are in fact used.

From a combined analysis of all the published ISL results, including contributions from Bfactories, CDF, CLEO, and DELPHI[5], the Heavy Flavor Averaging Group [6] quotes the results shown table 1 in in the so called "kinetic scheme"[7]; for sake of comparison the results obtained in the so called (1S) scheme [8], are also reported.

\section{3. $\left|V_{c b}\right|$ from ESL}

The decays $B \rightarrow \ell^{-} \bar{v}_{\ell} D^{(*)}$ are selected for both neutral and charged $\mathrm{B}$ mesons, where the $D^{*}$ is then reconstructed in the $D \pi$ decay mode. The $D^{0}$ is reconstructed into at most four possible final states $\left(K^{-} \pi^{+}\left(\pi^{0}\right), K_{s} \pi^{+} \pi^{-}, K^{-} \pi^{+} \pi^{+} \pi^{-}\right)$, the $D^{+}$into $K^{-} \pi^{+} \pi^{+}$and $K_{s} \pi^{+}$. The decay rate is expressed as the product of several terms [4]:

$$
\frac{d \Gamma}{d w}=\left|V_{c b}\right|^{2} \times \frac{G_{F}^{2} m_{B}^{5}}{48 \pi^{3}} \times(1-w)^{\zeta} \times \eta_{e w} \times \mathscr{P}(w) \times \mathscr{F}(w),
$$

where $w=E_{D^{(*)}}^{\prime} / m_{D^{(*)}}$ is the Lorentz boost of the $\mathrm{D}^{(*)}$ meson in the B rest frame, $\zeta=1.5$ for $\mathrm{D}$ and $\zeta=0.5$ for $\mathrm{D}^{*}, \mathscr{P}(\mathrm{w})$ is the phase space term, $\eta_{e w}=1.007 \pm 0.005$ [10] the contribution from electroweak corrections, and $\mathscr{F}(w)$ is a shorthand for the form factors, one per each different possible helicity mode of the decay (one for the pseudoscalar D final state and four for the $\mathrm{D}^{*}$ ). In the case of infinite masses of the $\mathrm{B}$ and $\mathrm{D}$ mesons, all form factors would reduce to the Isgur Wise function[2], with the normalization $\mathscr{F}(1)=1$. In facts, each form factor is parameterized as a power series of $(w-1)$. Higher order coefficients are expressed as a function of the first order term (the slope $\rho$ ) using bounds inspired by analicity and by Heavy Quark Effective Theory [9]. The normalization factors $\mathscr{F}(1)=1.054 \pm 0.004 \pm 0.008$ [12] and $\mathscr{F}^{*}(1)=0.906 \pm 0.013$ [13] 
are computed using lattice QCD for $\mathrm{D}$ and $\mathrm{D}^{*}$ final states ${ }^{1}$. Experiments fit the differential decay rates, extracting the normalization $\mathscr{F}^{(*)}(1) \times\left|V_{c b}\right|$ and the slope.
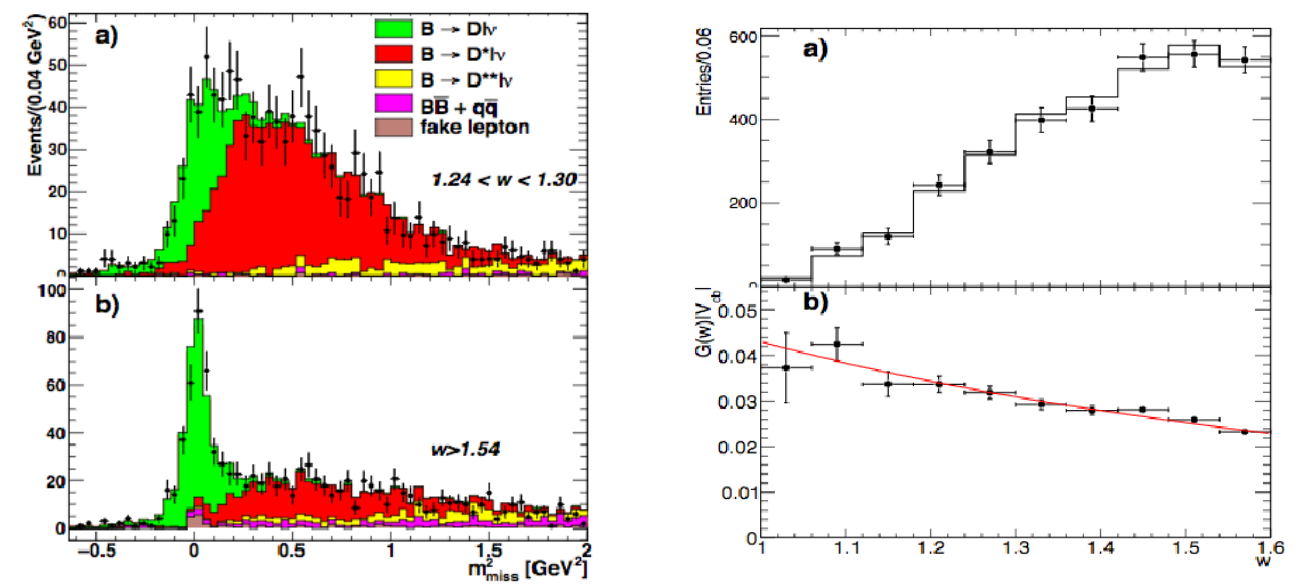

Figure 1: Left: squared missing mass in two $w$ bins from the tagged $B A B A R$ measurement of $B \rightarrow D \ell \bar{v}_{\ell}$. Green : signal, red, background from $\mathrm{D}^{*}$, yellow, background from higher $\mathrm{D}$ states. Right top: background subtracted event rate. Right bottom: the form factor, fitted with HQET inspired function.

$B A B A R$ [14] and Belle [15] have selected samples of 50 and 120 thousands of untagged decays to vector final states. The fit is extended to four dimensions, including the angles between the decay planes into which the event can be decomposed, in order to determine the coefficients related to all four helicity amplitudes. Both $B A B A R$ and Belle fit the four projected distributions and then account for correlations with parameterized ("toy") simulations. More recently, Belle has published a tagged analysis [16] consisting of about 2000 events. The kinematic constraints coming from the fully reconstructed B allow a more precise definition of $w$ and of the angles, partially compensating for the small data size. Unfolded spectra are provided, including correlations. All these analysis provide consistent results.

Although theoretically simpler, decays to scalar D meson are a tough experimental issue: by one side they receive a very large feed down background from $\mathrm{D}^{*}$ events where the soft pion (or the photon) is missed; by the other the extrapolation to $w=1$ is more difficult due to helicity suppression. For these reasons the most precise results are obtained by tagged analysis, where the $\mathrm{D}^{*}$ feed down can be significantly reduced using kinematic constraints to determine the missing mass in the event which, for signal events, corresponds to that of the neutrino. Fig.1 shows the results from the $B A B A R$ measurement.

Table 2 summarizes the results on $\left|V_{c b}\right|$ (and other significant parameters) obtained from exclusive semileptonic decays. The consistency among the experimental results is good both for vector and for pseudoscalar decays: after correcting for the end-point normalization factors, also the values of $\left|V_{c b}\right|$ are consistent, as shown in Tab.2.

It should however be noted that these values are barely compatible with the inclusive result, reported in table 1.

\footnotetext{
${ }^{1}$ Symbols are chosen for clarity and do not correspond to the usual bibliographic conventions
} 
Table 2: HFLAV averages for the $\left|V_{c b}\right|$ measurements from ESL decays. As for $\left|V_{c b}\right|$ the first error is experimental, the second theoretical. For the other parameters the total error is reported.

\begin{tabular}{l|c|c|c|c|c}
\hline Mode & $\left|V_{c b}\right| \times 10^{3}$ & $\rho^{2}$ & $R_{1}(1)$ & $R_{2}(1)$ & $\chi^{2} /$ dof \\
\hline $\mathrm{D}$ & $39.18 \pm 0.94 \pm 0.36$ & $1.128 \pm 0.033$ & - & - & $4.7 / 8$ \\
$\mathrm{D}^{*}$ & $38.71 \pm 0.47 \pm 0.59$ & $1.205 \pm 0.026$ & $1.404 \pm 0.032$ & $0.854 \pm 0.020$ & $30.2 / 23$ \\
\hline
\end{tabular}

Triggered by this longstanding inconsistency, the authors in [17] have fitted Belle $\mathrm{D}^{*}$ unfolded data relaxing the constraints from HQET which bound the higher order parameters to the slope $\rho$. Their result, $\left|V_{c b}\right|=(41.7 \pm 2$. $) \times 10^{-3}$ is sizably larger and now consistent with the inclusive determination. It has been observed, however, that the values of the higher order parameters are unexpected, suggesting "possibly large deviations from Heavy Quark Symmetry" [18].

\section{4. $\left|V_{u b}\right|$ from ISL decays}

The main issue when dealing with $b \rightarrow u$ transitions is the large feed down background from $b \rightarrow c$ decays, with a typical signal to noise ratio of about $2 \%$. To cope with that, profiting of the much smaller mass of the $u$ quark wrt the $c$, measurements are performed in regions of the phase space out of reach for $b \rightarrow c$ decays. This approach has however several drawbacks: due to resolution effects charm background nevertheless leaks in the signal region, the statistical significance of the signal is heavily reduced, and, what is worse, QCD inspired models are needed to compute the large fraction of signal rejected by the selection, so introducing a sizable model dependence. Traditional untagged analysis rely on selecting electrons with momentum larger than that allowed for $b \rightarrow \ell^{-} \bar{v}_{\ell} c$ decays (about $2.3 \mathrm{GeV}$ ) reducing to less than $10 \%$ the observed phase space. Further information can be gathered in tagged analysis, reconstructing the visible hadronic mass, and (or) the square of the momentum transferred to the two leptons $q^{2}=\left(p_{\ell}+p_{v}\right)^{2}=\left(p_{B}-p_{X u}\right)^{2}$ (corresponding to the square of the mass of the virtual $\mathrm{W}$ boson). In such a way, looser cuts can be applied so reducing somewhat the uncertainty introduced by the extrapolation. To increase the confidence in the results, measurements are performed selecting different phase space regions and using several different models inspired by HQET, with parameters tuned from the inclusive $\left|V_{c b}\right|$ fits. Consistent results are obtained from a dozen of different measurements. The world average value is computed from the HFLAV [6] using the GGOU model [19] :

$$
\left|V_{u b}\right|=\left(4.52 \pm 0.27_{-0.14}^{+0.11}\right) \times 10^{-3}
$$

where the first error is experimental and the second theoretical. Most of the other models considered are consistent within few parts per cent [20].

\section{5. $\left|V_{u b}\right|$ from ESL decays}

Even if several decay modes have been considered in the past, the most precise measurements come from the process $B \rightarrow \ell \bar{v}_{\ell} \pi$. The decay width is measured as a function of $q^{2}$. The unique form factor is expressed by a power series expansion with four free parameters, including $\left|V_{u b}\right|$ 
[21]. Bounds from Lattice QCD at large $q^{2}$ and from Light Cone Sum Rules [23] are imposed to ease the fit convergence. Measurements exploit neutrino reconstruction: in untagged analysis a coarse resolution is obtained by using the missing energy and missing momentum, ad assuming the B meson at rest; much better resolution is obtained in tagged analysis thanks to the sharp kinematic constraints. All in all the two approaches provide comparable precision, statistic abundance compensating for larger background and worse resolution. The result:

$$
\left|V_{u b}\right|=\left(3.65 \pm 0.09_{\text {exp }} \pm 0.11_{t h}\right) \times 10^{-3} .
$$

is more precise than the one obtained from ISL decays. As for $\left|V_{c b}\right|$ the two measurements are barely compatible.
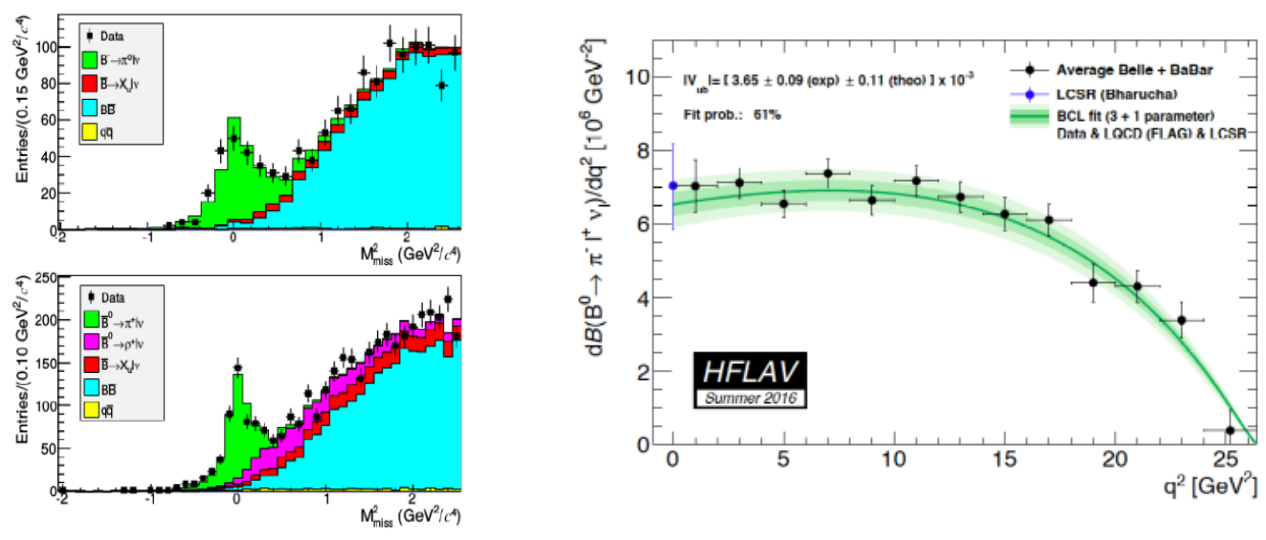

Figure 2: Left: squared missing mass in the tagged Belle measurement of $B \rightarrow \pi \ell \bar{v}_{\ell}$ for $\mathrm{B}^{+}$(top), and $\mathrm{B}^{0}$ (bottom). Right: fit to the form factor performed by the HFLAV on Belle $+B A B A R$ data

\section{Measurement of $\mathscr{B}\left(B \rightarrow \tau^{-} \bar{v}_{\tau} D^{(*)}\right)$}

As compared to the light lepton case, this process is an experimental nightmare: the $\tau$ decays to several different final states, all implying the production of a neutrino, which prevents the full reconstruction of the heavy lepton. Two or even three neutrinos are produced in the process, loosening the bounds from kinematic constraints even in tagged events. Finally, due to the larger $\tau$ mass, ad-hoc form factors must be used to describe the decays.

Not surprisingly, the first measurements of this decay were performed only at B-factories[24],[25]. Measurements exploit tagged event, focusing only on the leptonic decays $\tau \rightarrow \ell \bar{v}_{\ell} v_{\tau}$. The main source of background, $B \rightarrow \ell^{-} \bar{v}_{\ell} D^{(*)} X$ decays, is separated from the signal on topological basis; signal events are characterized by larger values of the squared missing mass $\left(M_{\text {miss }}^{2}\right)$, smaller lepton momentum $\left(p_{\ell}\right)$, smaller residual energy after subtracting the contributions from the fully reconstructed B and the particles assigned to the $D^{(*)} \tau$ candidate. BABAR performs a $2 \mathrm{D}$ fit in the $M_{\text {miss }}^{2}$, $p_{\ell}$ plane. Belle normalizes the background in the signal depleted region defined by $M_{\text {miss }}^{2}<0.8$ and then fits the signal contribution using a multivariate tag combining the most discriminant variables in the complementary $M_{m i s s}^{2}$ region. Both experiments fit simultaneously D and $\mathrm{D}^{*}$ final states in 
order to properly deal with the feed down from the second to the first (see Fig.3. In addition, Belle has also performed two other analysis of the $\mathrm{D}^{*}$, one looking at hadronic $\tau$ final state and the other exploiting a semileptonic tag[26]. To reduce experimental and theoretical uncertainties, experiments measure the ratios of branching fractions $R\left(D^{(*)}\right)=\frac{\mathscr{B}\left(B \rightarrow \tau^{-} \bar{v}_{\tau} D^{(*)}\right)}{\mathscr{B}\left(B \rightarrow \ell^{-} \bar{v}_{\ell} D^{(*)}\right)}$, separately for $\mathrm{D}$ and $\mathrm{D}^{*}$ final states. In addition to the above, Belle has performed two other measurements of $R\left(D^{*}\right)$ only, one using hadronic $\tau$ decays, the other exploiting fully reconstructed semileptonic B decays on the tag side.
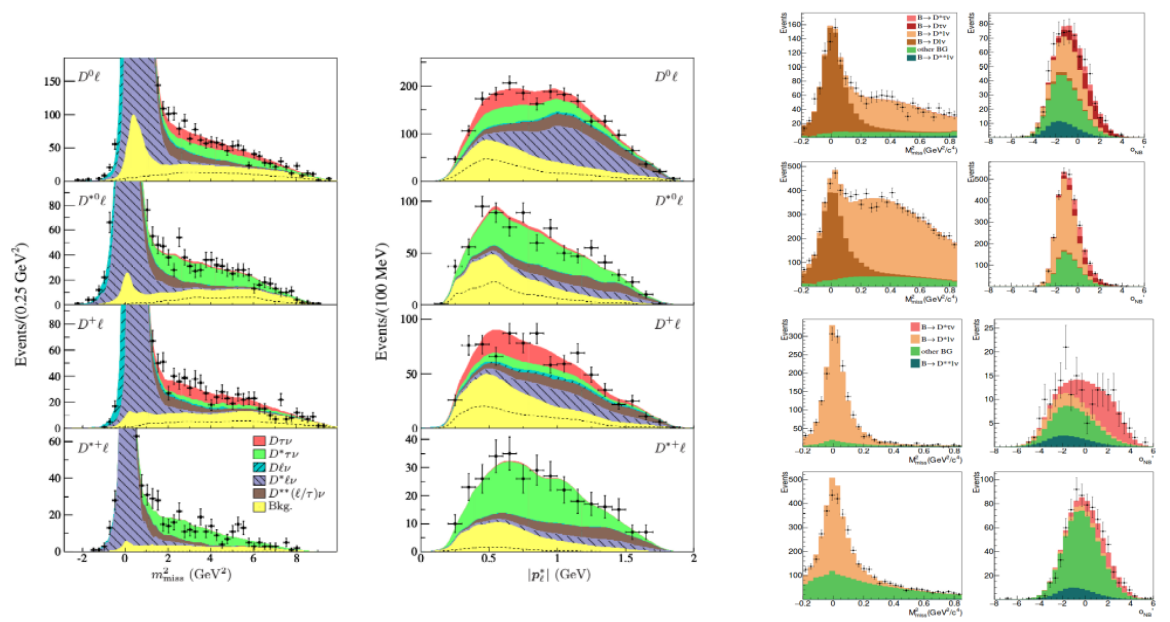

Figure 3: Left: projections along $M_{m i s s}^{2}$ (first column) and $p_{\ell}$ (second column) from the $B A B A R$ analysis. Right: $M_{m i s s}^{2}$ and BDT output for events with $M_{\text {miss }}^{2}>0.8$ from the Belle analysis

Results are reported in Fig.4 which compares the individual measurements (including $\mathrm{LHCb}$ contributions), the HFLAV average and the prediction of the standard model: the discrepancy between the measurements and the expectation amounts to about four standard deviations.

\section{Conclusions}

The study of semileptonic B decays allows a detailed understanding of $b \rightarrow c$ and $b \rightarrow u$ transitions. The large amount of data collected at the B-factories, the high quality of the detectors and the use of sophisticated analysis tools push the experimental precision to the percent level; steady progresses in computation techniques (Heavy Quark Symmetry, OPE and Lattice QCD), and the interplay between theory and experiment, allowed comparable precision in the interpretation of the resuts, so that nominal uncertainties of $\mathscr{O}(1 \%)$ and $\mathscr{O}(5 \%)$ are achieved for $\left|V_{c b}\right|$ and $\left|V_{u b}\right|$ . That notwithstanding (or maybe, as a consequence) several long-standing problems have not yet been solved, like the inconsistencies between the inclusive and exclusive determinations of both $\left|V_{u b}\right|$ or $\left|V_{c b}\right|$. Attemps to solve at least in part this puzzle [17], clash with the expectations of HQS [18]. On the experimental side several improvements are still possible. As for $\left|V_{u b}\right|$, more effort should be put in understanding the $b \rightarrow c$ background leaking in the inclusive $b \rightarrow u$ samples; measurements should be performed with a consistent use of the models for the signal and the background. As for $\left|V_{c b}\right|$ fits to the large data sets of $B \rightarrow \ell^{-} \bar{v}_{\ell} D^{(*)}$ collected by B-factories 


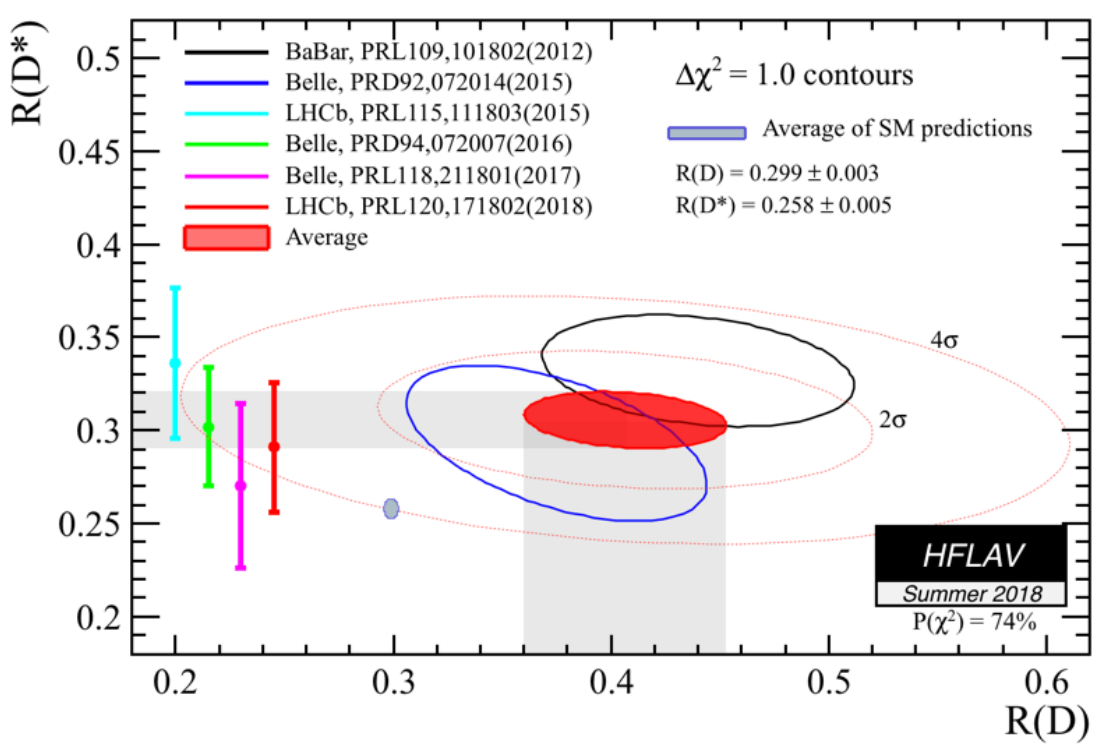

Figure 4: Contour plots in the $\mathrm{R}(\mathrm{D}) \mathrm{R}\left(\mathrm{D}^{*}\right)$ plane. The red area shows the $1 \sigma$ contour from the HFLAV average (and, dashes, the 2 and $4 \sigma$ ) and the standard model prediction (gray ellipse). Individual contributions are also displayed.

should be repeated relaxing the constraints on the high order coefficients in order to test HQET. The background from higher mass charm states (orbital and radial excitations) should be better controlled with dedicated analysis (this might also clarify the old issue that the sum of the branching fraction of the observed charm final states does not add to the inclusive branching fraction); as higher charm excitations also constitute a sizable background for the $B \rightarrow \tau^{-} \bar{v}_{\tau} D^{(*)}$ analysis, this might also increase confidence in the $B \rightarrow \tau^{-} \bar{v}_{\tau} D^{(*)}$ anomaly, or else reduce its significance.

\section{References}

[1] M.A. Shifman and M.B. Voloshin, Sov. J. Nucl. Phys. 47, 511 (1988)

[2] N. Isgur and M.B. Wise, Phys. Lett. B232, 113 (1989); ibid. B237, 527 (1990).

[3] D. Benson et al., Nucl. Phys. B665, 367 (2003).

[4] See R. Kowalewski and T. Mannel in C. Patrignani et al. (Particle Data Group),Chin. Phys. C 40, 100001 (2016)

[5] B. Aubert et al. (BABAR Collab.), Phys. Rev. D69, 111103 (2004); B. Aubert et al. (BABAR Collab.), Phys. Rev. D69, 111104 (2004); C. Schwanda et al. (Belle Collab.), Phys. Rev. D75, 032005 (2007); P. Urquijo et al. (Belle Collab.), Phys. Rev. D75, 032001 (2007); S.E. Csorna et al. (CLEO Collab.), Phys. Rev. D70, 032002 (2004); A.H. Mahmood et al. (CLEO Collab.), Phys. Rev. D70, 032003 (2004); D. Acosta et al. (CDF Collab.), Phys. Rev. D71, 051103 (2005); J. Abdallah et al. (DELPHI Collab.), Eur. Phys. J. C45, 35 (2006).

[6] Amhis, Y. et al.,the Heavy Flavor Averaging Group,"Eur. Phys. J.,C77,895 (2017)", 
[7] A.Alberti et al., Phys.Rev.Lett. 114 (2015), 061802.

[8] A.H. Hoang et al., Phys. Rev. D59, 074017 (1999). updated results and plots available at https://hflav.web.cern.ch

[9] See C.W.Bauer and M.Neubert in C. Patrignani et al. (Particle Data Group), Chin. Phys. C 40, 100001 (2016)

[10] A. Sirlin, Nucl. Phys. B196, 83 (1982)

[11] C.G. Boyd, B. Grinstein, and R.F. Lebed, Phys. Rev. Lett. 74, 4603 (1995); ibid.Phys. Rev. D56, 6895 (1997); B. Grinstein and A. Kobach, Phys. Lett. B771, 359 (2017); I. Caprini,L.Lellouch,M.Neubert, Nucl. Phys. B530, 153 (1998).

[12] J. Bailey et al. [Fermilab Lattice and MILC Collab.], Phys. Rev. D92, 034506 (2015).

[13] J.A. Bailey et al. [Fermilab Lattice and MILC Collab.], Phys. Rev. D89, 114504 (2014)

[14] B. Aubert et al. (BABAR Collaboration) Phys. Rev. D 77, 032002, 2008.

[15] W. Dungel et al. (The Belle Collaboration) Phys. Rev. D 82, 112007, 2010.

[16] A.Adesellam et al. (the Belle Collaboration) arXiv:1702.01521 [hep-ex].

[17] B. Grinstein and A. Kobach, Phys. Lett. B771, 359 (2017); D. Bigi, P. Gambino and S. Schacht, Phys. Lett. B769, 441 (2017).

[18] F.U. Bernlochner et al.,Phys. Rev. D 96, 091503(R),2017.

[19] P. Gambino,et al., JHEP 0710:058,2007

[20] B.O. Lange, M. Neubert and G. Paz, Phys. Rev. D72:073006 (2005); J.R. Andersen and E. Gardi, JHEP 0601:097 (2006); U. Aglietti, et al.,EPJC, Vol. 59 (2009); C.W. Bauer, Z. Ligeti and M.E. Luke, Phys. Rev. D64:113004 (2001).

[21] C.Bourrely, L.Lellouch, and I.Caprini, Phys. Rev. D 79, 013008 (2008).

[22] J. A. Bailey et al., (FNAL/MILC Collab.) Phys. Rev. D92 (2015).

[23] A. Bharucha, JHEP 1205 (2012) 092.

[24] J.P.Lees et al. (BABAR Collab.) Phys.Rev.Lett. 109,101802 (2012); J.P.Lees et al. (BABAR Collab.) Phys.Rev.D 88, 072012 (2013)

[25] M. Huschle et al. (Belle Collaboration) Phys. Rev. D 92, 072014 , (2015)

[26] Y. Sato et al. (Belle Collaboration) Phys. Rev. D 94, 072007, (2016); S. Hirose et al. (Belle Collaboration) Phys. Rev. Lett. 118, 211801 (2017). 